\title{
MiR-125b Inhibits Tumor Growth and Promotes Apoptosis of Cervical Cancer Cells by Targeting Phosphoinositide 3-Kinase Catalytic Subunit Delta
}

Fang Cuia,c Xiuli Lib,c Xiangyu Zhu ${ }^{\mathrm{a}}$ Lili Huang ${ }^{\mathrm{a}}$ Yongfang Huanga ${ }^{\mathrm{a}}$ Caiying Mao ${ }^{\mathrm{a}}$ Qi Yan ${ }^{\mathrm{a}}$ Jianhong Zhu ${ }^{\mathrm{a}}$ Wenxia Zhao ${ }^{\mathrm{a}}$ Hong Shi $^{\mathrm{a}}$

aDepartment of Gynaecology and Obstetric Medicine, Shanghai Jiangwan Hospital, Shanghai; bDepartment of Dermatology, Shanghai 10th People's Hospital, Tongji University School of Medicine, Shanghai; 'These authors contributed equally to the study

\section{Key Words}

Phosphoinositide 3-kinase catalytic subunit delta - Post-transcriptional regulation - Cervical cancer $\cdot$ HeLa $\cdot$ Apoptosis

\begin{abstract}
Backgroud: microRNAs (miRNAs) are involved in cancer-related processes. The miRNA-125b (miR-125b) has been identified as miRNA over-expressed in a wide variety of cancers. However, the role of miR-125b in the context of cervical carcinoma remains unknown. Methods: In this study, the effect of miR-125b on the proliferation and apoptosis of human cervical cells was analyzed by MTT assay and Flow cytometry analysis. we identified phosphoinositide 3-kinase catalytic subunit delta (PIK3CD) as a novel miR-125b target. Results: overexpression of miR$125 \mathrm{~b}$ in HeLa cervical cancer cells decreased cell proliferation, induced apoptosis and downregulated expression of PIK3CD. To identify the mechanisms responsible, we investigated the PI3K/Akt pathway and found that PI3K, phospho-Akt, and phospho-mTOR were all downregulated, while Bid was up-regulated in miR-125b-overexpressing subclones. In vivo, over expression of miR-125b in HeLa cells markedly reduced their ability to form tumors. Conclusion: these results suggest that miR-125b suppresses tumor growth activity by targeting the PI3K/ Akt/mTOR signal-ing pathway, and may provide a target for effective therapies.
\end{abstract}

Copyright (C) 2012 S. Karger AG, Basel 


\section{Introduction}

Cervical cancer is a major problem worldwide and a leading cause of cancer death in women. Most cervical cancer patients receive standard radiotherapy and chemotherapy. However, clinical outcomes vary dramatically and are difficult to predict. It was estimated that there would be about 13,570 new cases of cervical cancer in the United States in 2011, and 4,650 related deaths (statistics provided by the American Cancer Society). It is therefore vital to find new, effective treatment approaches to reduce mortality and extend patient survival.

MicroRNAs (miRNAs) are a family of small non-coding RNA molecules that downregulate the expression of their protein-coding target genes [1]. These RNAs are 19-25 nucleotides (nt) long and are cleaved from 70-100-nt hairpin pre-miRNA precursors. miRNAs are considered to be master regulators of many important biological processes, including apoptosis, cell growth, viral infection and cancer development [2-5]. However, despite numerous studies, the role of miRNAs in cervical cancer remains poorly understood [6-12], and few studies have attempted a full characterization of the complex relationships between miRNAs and their target mRNAs in cervical malignant transformation [13-19].

miRNA-125b confers temozolomide resistance to human glioblastoma stem cells through the mitochondrial pathway of apoptosis [19]. Wilting et al. found that altered expression levels of five significantly differentially-expressed miRNAs, hsa-miR-9 (1q23.2), hsa-miR-15b (3q25.32), hsa-miR-28-5p (3q27.3), hsa-miR-100 and hsa-miR-125b (both 11q24.1), were directly linked to common chromosomal alterations [13, 20], while Zhao et al. found that miR-125b acted as a tumor suppressor in hepatic tumor development by targeting Bcl-2 and inducing cancer cell apoptosis [13]. These findings suggest that miR$125 \mathrm{~b}$ may also be associated with cervical cancer progression through the regulation of PIK3CD expression. The phosphoinositide 3-kinase PI3K/Akt/mTOR pathway is known to be associated with cell proliferation and apoptosis. Previous studies have indicated that overexpression of miR-7 inhibits the Akt pathway in hepatocellular carcinoma (HCC) [21]. We therefore investigated the ability of miR-125b to regulate cervical cancer growth and apoptosis through interactions with the PI3K/Akt/mTOR pathway.

The PI3K catalytic subunit delta (PIK3CD) participates in regulating the PI3K pathway and is also involved in the development and progression of HCC [22], breast cancers [23] and glioma cells [24]. However, its role in cervical carcinogenesis remains unknown.

In the present study, we therefore tested the hypothesis that miR-125b could inhibit tumor growth and promote apoptosis of cervical cancer by interacting with the PI3K/Akt/ mTOR pathway and by targeted regulation of PIK3CD expression.

\section{Materials and Methods}

\section{Cell transfection and miRNA transfection}

HeLa cells were acquired from the American Type Culture Collection and cultured in Dulbecco's Modified Eagle's Medium (Gibco) supplemented with 10\% fetal bovine serum, 100 units/ml penicillin, $100 \mu \mathrm{g} / \mathrm{ml}$ streptomycin, $2 \mathrm{mM}$ glutamine, and $0.1 \mathrm{mM}$ non-essential amino acids solution at $37^{\circ} \mathrm{C}$ in a humidified chamber supplemented with 5\% CO2. MiRNAs and constructed vectors were transfected using Lipofectamine 2000 (Invitrogen) according to the manufacture's instruction. The miR-125b mimic and a nonspecific miR control were all purchased from Sigma Company.

\section{Cell proliferation assay}

For cell proliferation assays, HeLa cells were seeded in 96-well plates at 7000 cells/well and transfected with negative control $(50 \mathrm{nM})$ or miR-125b precursor $(50 \mathrm{nM})$. Cell growth was measured at indicated times using the Cell Titer 96 Non-Radio- active Cell Proliferation Assay (Promega). 


\begin{tabular}{|c|c|c|}
\hline Cellular Physiology & Cell Physiol Biochem 2012;30:1310-1318 & \\
\hline and Biochemistry & $\begin{array}{l}\text { DOI: } 10.1159 / 000343320 \\
\text { Published online: October 22, } 2012\end{array}$ & $\begin{array}{l}\text { O } 2012 \text { S. Karger AG, Basel } \\
\text { www.karger.com/cpb }\end{array}$ \\
\hline
\end{tabular}

Colony-formation assay

HeLa cells were transfected with negative control ( $50 \mathrm{nM}$ ) or miR-125b precursor (50 nM) in 6-well plates at a density of $2 \times 10^{6}$ cells/well. Cells were subsequently trypsinized after $24 \mathrm{~h}$, and seeded in 5 -cm culture dishes (1000 cells/well) to form colonies. After 10 days, colonies were fixed in $4 \%$ paraformaldehyde and stained in $0.1 \%$ crystal violet solution.

Con truction of the miR-125b Expression Vector

Oligonucleotides for the preparation of the miR-125b duplexes were ordered from Integrated DNA Technologies (IDT, Coral-ville, IA). The following sequences of oligonucleotides were used: miR-125b forward/HindIII, 5'-GGCAAGCTTAACATTGTTGCGCTCCTCTCA-3'; miR-125b reverse/Bam HI, 5' -TATGGATCCTTCCAGGATGCAAAAGCACGA-3'. After being double digested with HindIII and BamHI, the PCR product was cloned into pSilencer 4.1-U6 neo vector (Ambion,Austin, TX). The resulting vector was named pSilencer/miR-125b. Expression of miR-125b was detected by qRT-PCR analysis after $48 \mathrm{~h}$ from transfection miR-125b and Neg-control plasmid into Hela for $48 \mathrm{~h}$.

Detection of apoptosis by flow cytometry (FCM)

Negative control and miR-125b precursor cells were trypsinized, collected, washed and then stained with annexin V-fluorescein isothiocyanate (BD Pharmingen) and propidium iodide (PI, Sigma) for $10 \mathrm{~min}$ at $4^{\circ} \mathrm{C}$, according to the manufacturer's protocol. Apoptotic cells were determined by FCM analysis (FACScan; Becton-Dickinson).

Reverse transcription-polymerase chain reaction (RT-PCR)

Total RNAs were extracted from cultured cells using TRIzol reagent, and reverse-transcribed into cDNA with SuperScript II reserve transcriptase (Invitrogen, Carlsbad, CA, USA). In brief, RT reactions were performed using the High Capacity cDNA Reverse Transcription Kit (Applied Biosystems). For RT-PCR, the primer sequences of various genes were demen-strated as follows: GAPDH: 5' -AAGGTCGGAGTCAACGGATT-3', and 5'-CTGGAAGATGGTGATGGGATT-3'; PIK3CD: 5'-TCTGCTAGCACATTGGTCCTAAAGGGGCT-3' and 5'-GGTGATATCGTTTCCCTTCGCAGATGTGT-3'. Each RT reaction included $150 \mathrm{ng}$ RNA as the template and a pool of RT primers. Real-time PCR was performed with Power SYBR Green PCR Master Mix (Applied Biosystems). All reactions were run in triplicate. All experiments were repeated three times.

\section{Luciferase assay}

HeLa cells were seeded in 24-well plates $\left(6 \times 10^{4} /\right.$ well). After $24 \mathrm{~h}$, cells were co-transfected with pGL3 vector containing the 3'UTR fragment or 3'UTRmut fragment of PIK3CD, Renilla vector (pRL-TK) and pcDNA6.2-miR-125b or pcDNA6.2-miR-Neg. Luciferase activities were measured $48 \mathrm{~h}$ post-transfection using the Dual-Luciferase Reporter Assay System (Promega). Luciferase activity was normalized to Renilla luciferase expression for each sample.

\section{Western blotting analysis}

Cells were lysed with RIPA lysis buffer. Proteins were separated by sodium dodecyl sulfatepolyacrylamide gel electrophoresis, and transferred to polyvinylidene difluoride membranes (Millipore, Bedford, MA, USA). Blots were blocked and then probed with antibodies against PI3K (1:500 dilution; Santa Cruz Biotechnology, Santa Cruz, CA, USA), phospho-Akt (Ser473; 1:400 dilution; Santa Cruz Biotechnology), Akt (1:1000 dilution; Santa Cruz Biotechnology), phospho-mTOR (1:500 dilution; Cell Signaling Technology Inc., Beverly, MA, USA), mTOR (1:1000 dilution; Santa Cruz Biotechnology), Bid (1:500 dilution; Cell Signaling Technology Inc., Beverly, MA, USA). After washing, the blots were incubated with horseradish peroxidase-conjugated secondary antibodies and visualized using super enhanced chemiluminescence detection reagent (Amersham Biosciences, Piscataway, NJ, USA).

\section{Tumorigenicity assay in nude mice}

Athymic BALB/c nude mice (4-6 weeks old, 18-22 g body weight) were randomly assigned to one of two groups ( 8 mice per group). HeLa/miR-125b and HeLa/Neg-Ctrl cells were trypsinized and resuspended at final concentrations of $2 \times 10^{6}$ cells $/ 0.1 \mathrm{ml}$ in phosphate-buffered saline. Cells were injected subcutaneously into the right flanks of nude mice. Mice were sacrificed 3 weeks later for evaluation. Tumor 


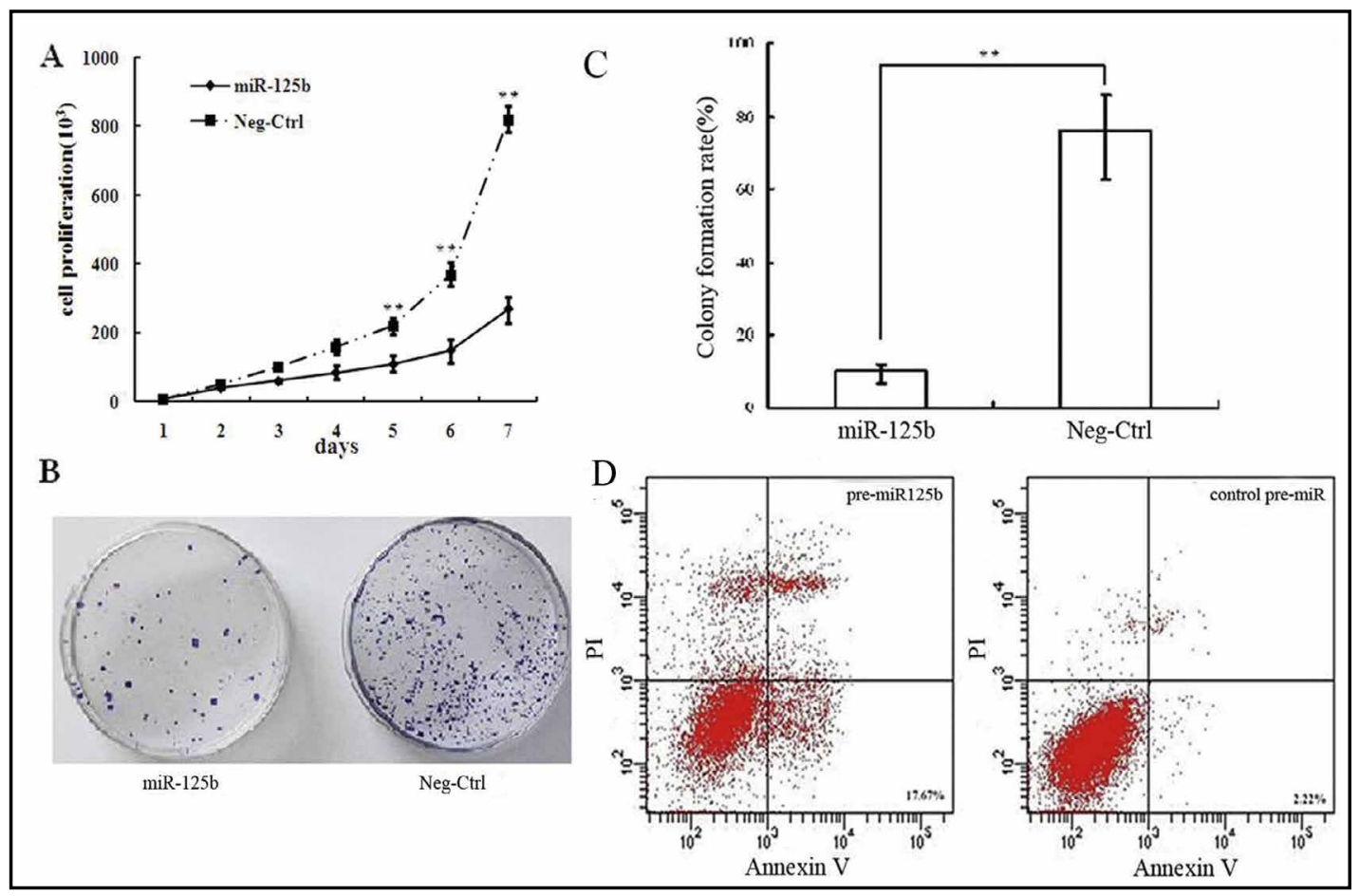

Fig. 1. Overexpression of miR-125b suppresses cell growth and colony formation. A: Cell proliferation assays. HeLa cells were transfected with miR-125b or Neg-Ctrl. Data are the average \pm s.d. of three independent experiments. ${ }^{* *} \mathrm{p}<0.01$ versus Ctrl. B: Colony-formation assays. C:Colony-formation rates were lower for miR-125b compared to Neg-Ctrl (about 80\% lower). D: Apoptotic rates of miR-125b-overexpressing and negative control groups, as measured by FCM-based Annexin V and PI binding analysis.

growth was monitored by measuring the largest (a) and smallest (b) perpendicular diameters with calipers, and calculating the tumor volume $(\mathrm{V})=\mathrm{a} \times \mathrm{b}^{2} \times 0.5$. Animal experiments were performed according to the institutional guidelines of the hospital committee on the use and care of animals.

\section{Statistical analysis}

Data are expressed as means \pm SD of three independent experiments, each performed in triplicate. Differences between groups were assessed by unpaired, two-tailed Student's t-test, $\mathrm{P}<0.05$ was considered significant.

\section{Results}

Overexpression of miR-125b suppressed proliferation and induced apoptosis of HeLa cells in vitro

Cell proliferation was significantly suppressed in HeLa cells transfected with miR-125b, compared to the negative control group (Fig. 1A). We also examined the effect of miR-125b on colony formation. HeLa cells transfected with miR-125b produced significantly fewer colonies (about 80\% fewer) than negative control cells (Fig. 1B and 1C).

To investigate the effect of miR-125b overexpression on apoptosis of HeLa cells, apoptotic cell death was assessed using an FCM-based Annexin V and PI binding assay. FCM analysis revealed apoptotic rates of $2.22 \% \pm 0.45 \%$, and $17.67 \% \pm 3.2 \%$ in the negative control and miR-125b-overexpressing groups, respectively $(\mathrm{P}<0.001$; Fig. 1D). Together, these results indicated that the inhibition of cell growth by miR-125b was associated with increased apoptosis. 


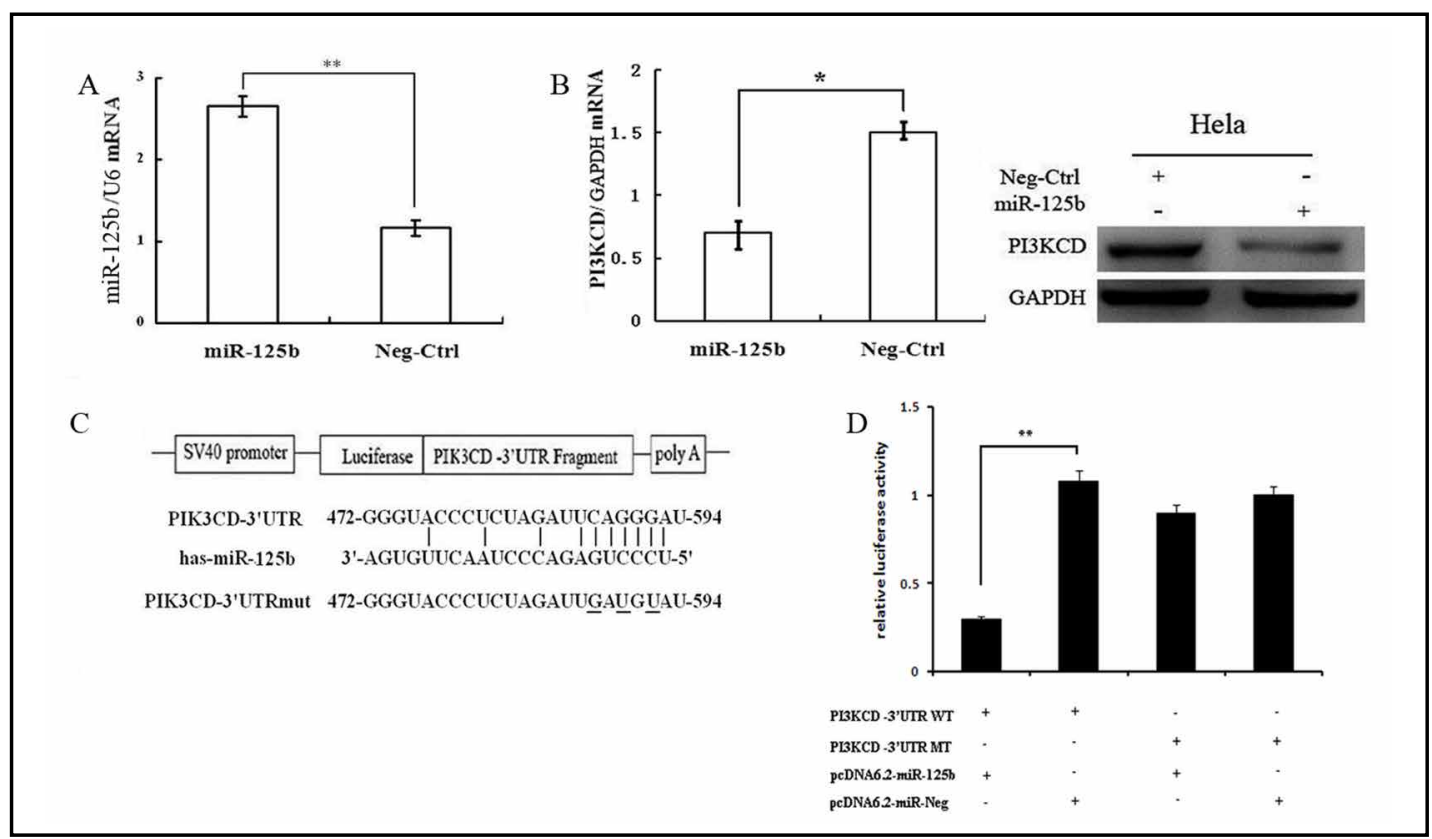

Fig. 2. Overexpression of miR-125b down-regulates PIK3CD expression in HeLa cells. A: Detection of miR$125 \mathrm{~b}$ or mature miR-125b by quantitative RT-PCR . B: Expression level of PIK3CD in HeLa cells transfected with miR-125b as detected by quantitative RT-PCR. Down-regulation of PIK3CD in HeLa cells transfected with miR-125b as detected by Western blot. miR-125b directly targets the PIK3CD-3'UTR. C: Diagram of PIK3CD -3'UTR-containing luciferase reporter gene construct and the PIK3CD-3'UTRmut fragment cloned from PIK3CD mRNA in HeLa cells. D: Luciferase reporter assays. Relative luciferase values normalized to co-transfections with miR-125b expression vector and PIK3CD-3'UTR or PIK3CD-3'UTRmut. Values are the average \pm s.d. of four replicates. ${ }^{* *} \mathrm{P}<0.01$.

PIK3CD is a target of miR-125b

miRNAs can regulate mRNA stability and the translation of target mRNAs at the posttranscriptional level [25]. Using TargetScan [26], we identified PIK3CD (3'UTR region to nucleotide positions 487-493) as a likely target of miR-125b. To further support the hypothesis that miR-125b regulates PIK3CD expression, we investigated endogenous PIK3CD mRNA and protein expression levels in HeLa cells transfected with miR-125b, using quantitative RT-PCR and Western blotting. The expression levels of miR-125b in HeLa cells, HeLa/ miR-125b and HeLa/Neg-Ctrl, were verified by quantitative RT-PCR ( $(\mathrm{P}<0.01$; Fig. 2A). Overexpression of miR-125b in HeLa cells resulted in significant reductions of PIK3CD mRNA and protein levels, compared to HeLa/Neg-Ctrl cells ( $\mathrm{P}<0.05$; Fig. 2B).

To investigate if the 3'UTR of PIK3CD acts as functional target of miR-125b, we performed an in vitro target reporter assay on HeLa cells expressing low levels of endogenous miR-125b. The 3'UTR of PIK3CD mRNA including the miR-125b putative binding site, or the mutant form in which the putative miR-125b binding site was mutated, was cloned downstream of the luciferase reporter gene (PIK3CD-3'UTR and PIK3CD-3'UTRmut). The specific interaction between miR-125b and PIK3CD is shown in Figure 2C. Transfection of either PIK3CD-3'UTR or PIK3CD-3'UTRmut alone into HeLa cells significantly inhibited the luciferase activities because of the function of endogenous miR-125b. However, co-transfection of the miR-125b expression vector with the wild-type PIK3CD-3'UTR reporter construct (PIK3CD-3'UTR), but not PIK3CD-3'UTRmut, resulted in a significant reduction in luciferase activity compared to the negative control group $(\mathrm{P}<0.05$; Fig. 2D). No significant differences in luciferase activities were found between the vehicle control and negative control groups. These results 


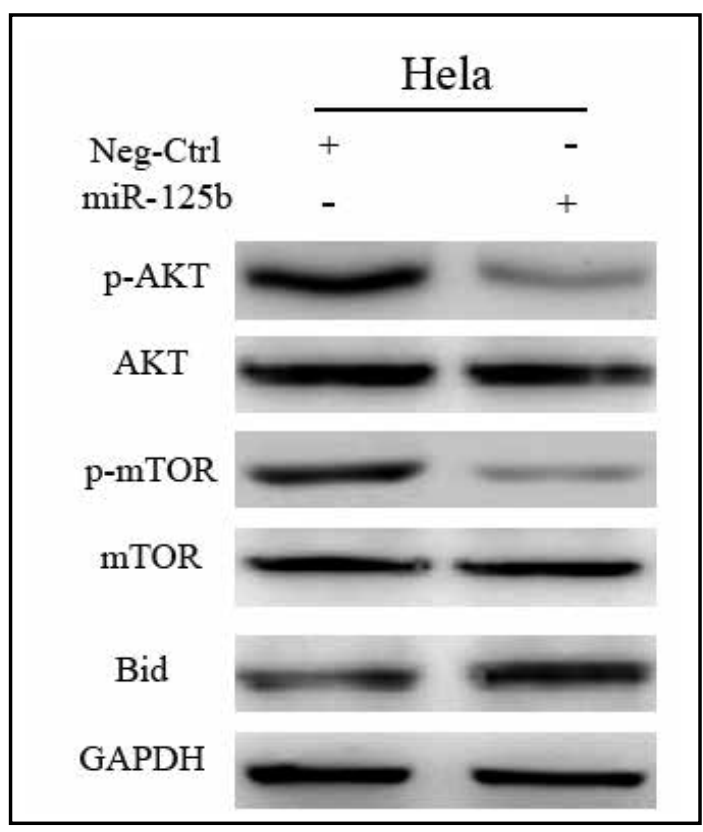

Fig. 3. Inhibition of PIK3CD expression by miR-125b alters the expression of key components in the PI3K/ Akt pathway. Overexpression of endogenous miR$125 \mathrm{~b}$ significantly reduced the levels of phosphoAkt and phospho-mTOR, whereas levels of the proapoptotic protein Bid were elevated in miR-125boverexpressing cells.

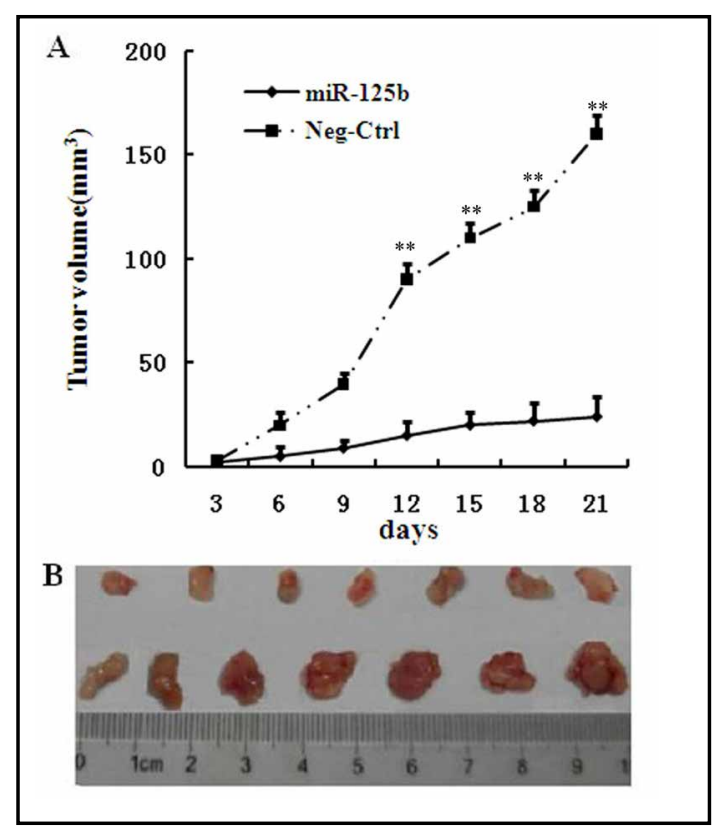

Fig. 4. miR-125b inhibits HeLa tumor cell growth in vivo. Tumor size was monitored every 3 days. Tumor volume was markedly increased in the negative control group on day 12 after tumor-cell inoculation, compared to the miR-125b-overexpressing group. A: Tumors volume was measured every 3 days after tumor cell implantation. B: HeLa/miR-125b and HeLa/Neg-Ctrl cells were injected subcutaneously into the right flanks of nude mice. Mice were sacrificed every 3 days for evaluation.

demonstrated that miR-125b directly and specifically interacted with PIK3CD mRNA and promoted PIK3CD protein silencing mostly through a post-transcriptional repression mechanism.

\section{miR-125b regulated PI3K/Akt/ mTOR pathway}

The protein expression levels of PI3K, total-Akt, phospho-Akt (p-Akt), mTOR (p-mTOR) were examined in negative control and miR-125b-overexpressing cells. Immunoblot analysis showed that overexpression of endogenous miR-125b significantly reduced the levels of PI3K, phospho-Akt, and phospho-mTOR, whereas the pro-apoptotic protein Bid was elevated in miR-125b-overexpressing cells (Fig. 3).

miR-125b inhibited tumor growth of HeLa cells in vivo

Given the suppression of HeLa-cell viability by miR-125b in vitro, we further evaluated if increased levels of miR-125b could inhibit the growth of HeLa cells in vivo. Tumor size was monitored every 3 days using calipers. Tumor volume in the negative control group was markedly increased on day 12 after tumor-cell inoculation, compared to the miR-125boverexpressing group (Fig. 4A; $\mathrm{P}<0.01$ ). Xenografts derived from miR-125b-overexpressing cells showed slow growth, compared to the negative control group $(\mathrm{P}<0.01)$, indicating that elevated miR-125b levels in HeLa cells markedly reduced their ability to form tumors (Fig. $4 \mathrm{~A}$ and $4 \mathrm{~B})$. 


\section{Discussion}

Cervical cancer is a complex disease involving the abnormal expression of many tumor suppressor genes and oncogenes. Recent studies have identified several genes associated with human cervical cancer. For example, expression of the pro-apoptotic protein Bax is often lost in carcinomas, and p53 has also been shown to play an important role in the development of cervical cancer [27]. Although focusing on known genes has yielded significant new information, previously unknown non-coding RNAs, including miRNAs, may also provide insights into the biology of cervical cancer.

miRNA expression signatures have been shown to be promising biomarkers for the classification or outcome prediction in a wide array of human cancers [28]. Genome-wide analyses have indicated that about half the miRNA genes are located at fragile sites and genomic regions with frequently dysregulated expression in cancers [29, 30]. Hu et al. [6] showed that both miR-200a and miR-9 could play important regulatory roles in cervical cancer, while other miRNAs and their target mRNAs have been implicated in cervical malignancies. Yao et al. [31] found that miR-21 played a potentially oncogenic role in the cellular processes of cervical cancer by targeting programmed cell death 4, while Qiang et al. [15] identified Plexin-B1 as a target of miR-214 in cervical cancer responsible for the growth and invasion of HeLa cells. GALNT7 has also been revealed as a target of miR-214, and has been shown to suppress growth and invasiveness of cervical cancer [32]. miR-143 is down-regulated in cervical cancer and promotes apoptosis and inhibits tumor formation by targeting Bcl-2 [14] .

miRNA-125b induces cancer-cell apoptosis through suppression of Bcl-2 expression [13]. Alpini et al. showed that epigenetic silencing of miR-125b contributed to an invasive phenotype in HCC, providing novel mechanistic insights and identifying a potential target mechanism that could be manipulated for therapeutic benefit [22]. In addition, Wang et al. showed that circulating miR-125b expression was associated with chemotherapeutic resistance in breast cancer [33]. These studies suggest that the role of miR-125b may differ among different cancer types.

The results of the present study demonstrate that miR-125b inhibits tumor growth and promotes apoptosis via down-regulation of PIK3CD in cervical cancer, both in vitro and in vivo. Luciferase assay identified PIK3CD as a special target of miR-125b, while cell proliferation and colony-formation assays showed that miR-125b suppressed cell proliferation. These results are supported by other recent studies on the mechanisms of miR-125b in HCC [22], breast cancer [33], and glioblastoma [19, 20]. Overexpression of miR-125b significantly induced apoptosis in cervical cancer cells; however, there are many possible mechanisms for inducing cell apoptosis. The results of this study demonstrated that the mechanism of apoptosis may involve miR-125b-mediated regulation of the PI3K/Akt/ mTOR pathway by targeting PIK3CD. Tumor volume in vivo decreased dramatically when miR-125b subclone cells were injected into mice, compared to the injection of control cells, further suggesting that the downregulation of PIK3CD by miR-125b may be involved in the pro-apoptotic function of miR-125b. The identification of PIK3CD as a miR-125b target gene may at least partially explain the molecular mechanisms of the tumor suppressor miR-125b.

In summary, this study provided the first evidence for the biological role of miR-125b in cervical cancer cells, and identified PIK3CD as a target potentially involved in miR-125bmediated growth suppression and apoptosis of cervical cancer cells via the PI3K/Akt/ mTOR pathway. These findings may provide the basis for the future use of miR-125b in the treatment of cervical cancer.

\section{Acknowledgements}

This study was funded by Traditional Chinese Medicine Scientific Research Funds from Shanghai Health Bureau (No. 2010L101A). 


\section{Cellular Physiology $\quad$ Cell Physiol Biochem 2012;30:1310-1318 and Biochemistry \\ Cui/Li/Zhu et al.: MiR-125b Inhibits PIK3CD in Cervical Cancer Cell}

\section{References}

1 Ambros V: The functions of animal micrornas. Nature 2004;431:350-355.

2 Miska EA: How micrornas control cell division, differentiation and death. Curr Opin Genet Dev 2005;15:563-568.

-3 Calin GA, Croce CM: Microrna signatures in human cancers. Nat Rev Cancer 2006;6:857-866.

-4 Johnson CD, Esquela-Kerscher A, Stefani G, Byrom M, Kelnar K, Ovcharenko D, Wilson M, Wang X, Shelton J, Shingara J, Chin L, Brown D, Slack FJ: The let-7 microrna represses cell proliferation pathways in human cells. Cancer Res 2007;67:7713-7722.

5 Kent OA, Mendell JT: A small piece in the cancer puzzle: Micrornas as tumor suppressors and oncogenes. Oncogene 2006;25:6188-6196.

-6 Hu X, Schwarz JK, Lewis JS, Jr., Huettner PC, Rader JS, Deasy JO, Grigsby PW, Wang X: Reduced mir-100 expression in cervical cancer and precursors and its carcinogenic effect through targeting plk1 protein 2011. Cancer Res 2011;70:1441-1448.

7 Lee JW, Choi CH, Choi JJ, Park YA, Kim SJ, Hwang SY, Kim WY, Kim TJ, Lee JH, Kim BG, Bae DS: Altered microrna expression in cervical carcinomas. Clin Cancer Res 2008;14:2535-2542.

-8 Lui WO, Pourmand N, Patterson BK, Fire A: Patterns of known and novel small rnas in human cervical cancer. Cancer Res 2007;67:6031-6043.

-9 Martinez I, Gardiner AS, Board KF, Monzon FA, Edwards RP, Khan SA: Human papillomavirus type 16 reduces the expression of microrna-218 in cervical carcinoma cells. Oncogene 2008;27:2575-2582.

10 Muralidhar B, Goldstein LD, Ng G, Winder DM, Palmer RD, Gooding EL, Barbosa-Morais NL, Mukherjee G, Thorne NP, Roberts I, Pett MR, Coleman N: Global microrna profiles in cervical squamous cell carcinoma depend on drosha expression levels. J Pathol 2007;212:368-377.

11 Pett MR, Herdman MT, Palmer RD, Yeo GS, Shivji MK, Stanley MA, Coleman N: Selection of cervical keratinocytes containing integrated hpv16 associates with episome loss and an endogenous antiviral response. Proc Natl Acad Sci U S A 2006;103:3822-3827.

12 Wang X, Tang S, Le SY, Lu R, Rader JS, Meyers C, Zheng ZM: Aberrant expression of oncogenic and tumorsuppressive micrornas in cervical cancer is required for cancer cell growth. PLoS One 2008;3:e2557.

13 Zhao A, Zeng Q Xie X, Zhou J, Yue W, Li Y, Pei X: Microrna-125b induces cancer cell apoptosis through suppression of bcl-2 expression. J Genet Genomics 2012;39:29-35.

-14 Liu L, Yu X, Guo X, Tian Z, Su M, Long Y, Huang C, Zhou F, Liu M, Wu X, Wang X: Mir-143 is downregulated in cervical cancer and promotes apoptosis and inhibits tumor formation by targeting bcl-2. Mol Med Report 2012;5:753-760.

15 Qiang R, Wang F, Shi LY, Liu M, Chen S, Wan HY, Li YX, Li X, Gao SY, Sun BC, Tang H: Plexin-b1 is a target of mir-214 in cervical cancer and promotes the growth and invasion of hela cells. Int J Biochem Cell Biol 2011;43:632-641.

-16 Wang F, Li Y, Zhou J, Xu J, Peng C, Ye F, Shen Y, Lu W, Wan X, Xie X: Mir-375 is down-regulated in squamous cervical cancer and inhibits cell migration and invasion via targeting transcription factor sp1. Am J Pathol 2011;179:2580-2588.

17 Xu J, Li Y, Wang F, Wang X, Cheng B, Ye F, Xie X, Zhou C, Lu W: Suppressed mir-424 expression via upregulation of target gene chk1 contributes to the progression of cervical cancer. Oncogene 2012; doi: 10.1038/onc.2012.121.

$>18$ Li BH, Zhou JS, Ye F, Cheng XD, Zhou CY, Lu WG, Xie X: Reduced mir-100 expression in cervical cancer and precursors and its carcinogenic effect through targeting plk1 protein. Eur J Cancer 2011 47:2166-2174.

19 Shi L, Zhang S, Feng K, Wu F, Wan Y, Wang Z, Zhang J, Wang Y, Yan W, Fu Z, You Y: Microrna-125b-2 confers human glioblastoma stem cells resistance to temozolomide through the mitochondrial pathway of apoptosis. Int J Oncol 2011;40:119-129.

20 Wilting SM, Snijders PJ, Verlaat W, Jaspers A, van de Wiel MA, van Wieringen WN, Meijer GA, Kenter GG, Yi Y, le Sage C, Agami R, Meijer CJ, Steenbergen RD: Altered microrna expression associated with chromosomal changes contributes to cervical carcinogenesis. Oncogene 2012; doi: 10.1038/onc.2012.20.

21 Li BH, Zhou JS, Ye F, Cheng XD, Zhou CY, Lu WG, Xie X: Reduced mir-100 expression in cervical cancer and precursors and its carcinogenic effect through targeting plk1 protein Eur J Cancer 2011;47:2166-2174. 


\section{Cellular Physiology $\quad$ Cell Physiol Biochem 2012;30:1310-1318 and Biochemistry \\ Cui/Li/Zhu et al.: MiR-125b Inhibits PIK3CD in Cervical Cancer Cell}

22 Alpini G, Glaser SS, Zhang JP, Francis H, Han Y, Gong J, Stokes A, Francis T, Hughart N, Hubble L, Zhuang SM, Meng F: Regulation of placenta growth factor by microrna-125b in hepatocellular cancer. J Hepatol 2011;55:1339-1345.

-23 Tang F, Zhang R, He Y, Zou M, Guo L, Xi T: Microrna-125b induces metastasis by targeting stard13 in mcf-7 and mda-mb-231 breast cancer cells. PLoS One 2012;7:e35435.

24 Xia HF, He TZ, Liu CM, Cui Y, Song PP, Jin XH, Ma X: Mir-125b expression affects the proliferation and apoptosis of human glioma cells by targeting bmf. Cell Physiol Biochem 2009;23:347-358.

25 Bartel DP: Micrornas: Genomics, biogenesis, mechanism, and function. Cell 2004;116:281-297.

26 Lewis BP, Burge CB, Bartel DP: Conserved seed pairing, often flanked by adenosines, indicates that thousands of human genes are microrna targets. Cell 2005;120:15-20.

27 Karlidag T, Cobanoglu B, Keles E, Alpay HC, Ozercan I, Kaygusuz I, Yalcin S, Sakallioglu O: Expression of bax, p53, and p27/kip in patients with papillary thyroid carcinoma with or without cervical nodal metastasis. Am J Otolaryngol 2007;28:31-36.

28 Jay C, Nemunaitis J, Chen P, Fulgham P, Tong AW: Mirna profiling for diagnosis and prognosis of human cancer. DNA Cell Biol 2007;26:293-300.

-29 Calin GA, Sevignani C, Dumitru CD, Hyslop T, Noch E, Yendamuri S, Shimizu M, Rattan S, Bullrich F, Negrini M, Croce CM: Human microrna genes are frequently located at fragile sites and genomic regions involved in cancers. Proc Natl Acad Sci USA 2004;101:2999-3004.

-30 Zhang L, Huang J, Yang N, Greshock J, Megraw MS, Giannakakis A, Liang S, Naylor TL, Barchetti A, Ward MR, Yao G, Medina A, O'Brien-Jenkins A, Katsaros D, Hatzigeorgiou A, Gimotty PA, Weber BL, Coukos G: Micrornas exhibit high frequency genomic alterations in human cancer. Proc Natl Acad Sci USA 2006;103:9136-9141.

-31 Yao Q, Xu H, Zhang QQ, Zhou H, Qu LH: Microrna-21 promotes cell proliferation and down-regulates the expression of programmed cell death 4 (pdcd4) in hela cervical carcinoma cells. Biochem Biophys Res Commun 2009;388:539-542.

-32 Peng RQ Wan HY, Li HF, Liu M, Li X, Tang H: Microrna-214 suppresses growth and invasiveness of cervical cancer cells by targeting udp-n-acetyl-alpha-d-galactosamine:Polypeptide n-acetylgalactosaminyltransferase 7. J Biol Chem 2012;287:14301-14309.

33 Wang H, Tan G, Dong L, Cheng L, Li K, Wang Z, Luo H: Circulating mir-125b as a marker predicting

chemoresistance in breast cancer. PLoS One 2012; 7:e34210. 